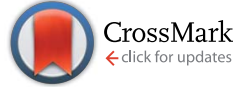

Cite this: RSC Adv., 2017, 7, 17387

Received 2nd December 2016 Accepted 7th March 2017

DOI: 10.1039/c6ra27674a

rsc.li/rsc-advances

\title{
Thin film transistors based on two dimensional graphene and graphene/semiconductor heterojunctions
}

\author{
Zhongcheng Zhu, $\uparrow^{\mathrm{a}}$ Imran Murtaza, $\dagger^{\mathrm{bc}}$ Hong Meng ${ }^{\star a b}$ and Wei Huang ${ }^{\mathrm{b}}$ \\ During the past few years, two-dimensional (2D) layered materials have emerged as the most fundamental \\ building blocks of a wide variety of optoelectronic devices. The weak van der Waals (vdW) interlayer forces \\ allow the 2D monolayers to isolate and restack into arbitrary stacking heterojunctions. The recently \\ developed chemical vapor deposition (CVD) technique shows great promise for the production of large \\ domain building blocks of 2D heterostructures with vertical and lateral stacking and much better device \\ performance. This review is the first of its kind to discuss the research progress of flexible FETs based on \\ graphene/semiconductor heterostructures, in which graphene acts as both electrode and semiconductor \\ material.
}

\section{Introduction}

Two-dimensional (2D) materials, such as nano-films, superlattices and quantum wells, refer to the materials in which the free movement (plane motion) of electrons takes place only in the two dimensional non-nanoscale. Graphene is the most significant among all the 2D materials proposed up till now ${ }^{\mathbf{1}}$ and has single (or several) atomic layer thickness. Beyond graphene, 2D materials also include transition metal oxides (e.g. $\mathrm{MnO}_{2}, \mathrm{ZnO}$ ), transition metal carbides and/or carbonitrides (MXene), transition metal chalcogenides (e.g. $\mathrm{MoS}_{2}$ ), hexagonal boron nitride (h-BN), topological insulators (e.g. $\mathrm{Bi}_{2} \mathrm{Se}_{3}, \mathrm{Sb}_{2} \mathrm{Te}_{3}$ ), silicene, germanene, black phosphorus (BP) and so on (Fig. 1). ${ }^{2-5}$ 2D materials as promising building blocks for novel nanoelectronics include semiconductors, insulators, transparent conductors and transducers, ${ }^{6-8}$ because amongst other considerations, these atomic sheets afford the ultimate thickness and scalability, which is desired in a variety of essential material categories. 2D semiconductors exhibit high carrier mobility, high on-off current ratio and excellent bendability that suit for future low-power consumption and flexible electronics. ${ }^{9-12}$ These materials are laterally composed of strong covalent bonds, which provide great in-plane stability. On the other hand, the weak van der Waals (vdW) interlayer forces allow us to isolate $2 \mathrm{D}$ monolayer and restack them into arbitrary stacking heterojunctions without the need to consider the

${ }^{a}$ School of Advanced Materials, Peking University Shenzhen Graduate School, Peking University, Shenzhen, 518055, China.E-mail: menghong@pkusz.edu.cn

${ }^{b}$ Institute of Advanced Materials, Nanjing Tech University, Nanjing 211816, China

${ }^{c}$ Department of Physics, International Islamic University, Sector H-10, Islamabad 44000, Pakistan

$\dagger$ First co-authors with equal contribution. atomic commensurability in contrast to their bulk counterparts. van der Waals (vdW)-bonded layered materials offer great variety in both structural and electronic properties, opening up new avenues for scientific research to obtain better device design and desired device functionalities by assembling heterostructures based on distinct 2D layers. Although, all-2D vdW heterostructures offer unique properties and functionalities compared with conventional devices, it still remains a challenge to produce entire families of $2 \mathrm{D}$ materials and their heterostructures over large areas with high electronic quality. Furthermore, control of doping type, carrier concentration and stoichiometry is quiet difficult for most 2D materials, limiting the scope and progress of all-2D vdW heterostructures. ${ }^{13}$

Graphene is the first truly $2 \mathrm{D}$ crystalline material, which is composed of periodic honeycomb lattice formed by $\mathrm{sp}^{2}$ hybrid orbitals of covalently bonded carbon atoms (Fig. $1 \mathrm{~b}$ and c). ${ }^{\mathbf{1 2 1 4 - 1 7}}$ The $\mathrm{p}$ orbital of coplanar carbon atoms overlap each other and form large conjugated $\pi$ bonds. There is a freely moving electron at each grid point, which endow graphene to have super high electron mobility $\left(2 \times 10^{5} \mathrm{~cm}^{2} \mathrm{~V}^{-1} \mathrm{~s}^{-1}\right)$ at low temperature $(\sim 5 \mathrm{~K}) .{ }^{\mathbf{1 , 1 8}}$ Graphene is theoretically considered as zero bandgap semiconductor and the electronic conduction velocity and theoretical resistivity of graphene are $8 \times 10^{5} \mathrm{~m} \mathrm{~s}^{-1}$ (ref. 19) and $10^{-6} \Omega \mathrm{cm}^{-1},^{20}$ respectively. Excellent electrical property of graphene favors the development of graphene based field effect transistors $^{21}$ and the supercapacitors. ${ }^{22}$ In addition, graphene has high optical transparency ${ }^{23}$ and has broad applications in the transparent conductive thin film solar cells, photon detectors and so on, ${ }^{\mathbf{2 4 - 2 8}}$ due to its unique optical properties. In addition, graphene also exhibits ultrahigh mechanical strength

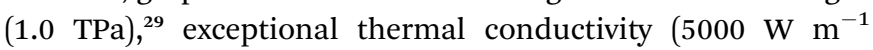
$\left.\mathrm{K}^{-1}\right),{ }^{30}$ half-integer quantum Hall effect at room temperature, ${ }^{31}$ Klein tunneling effect, ${ }^{32}$ and so on. 

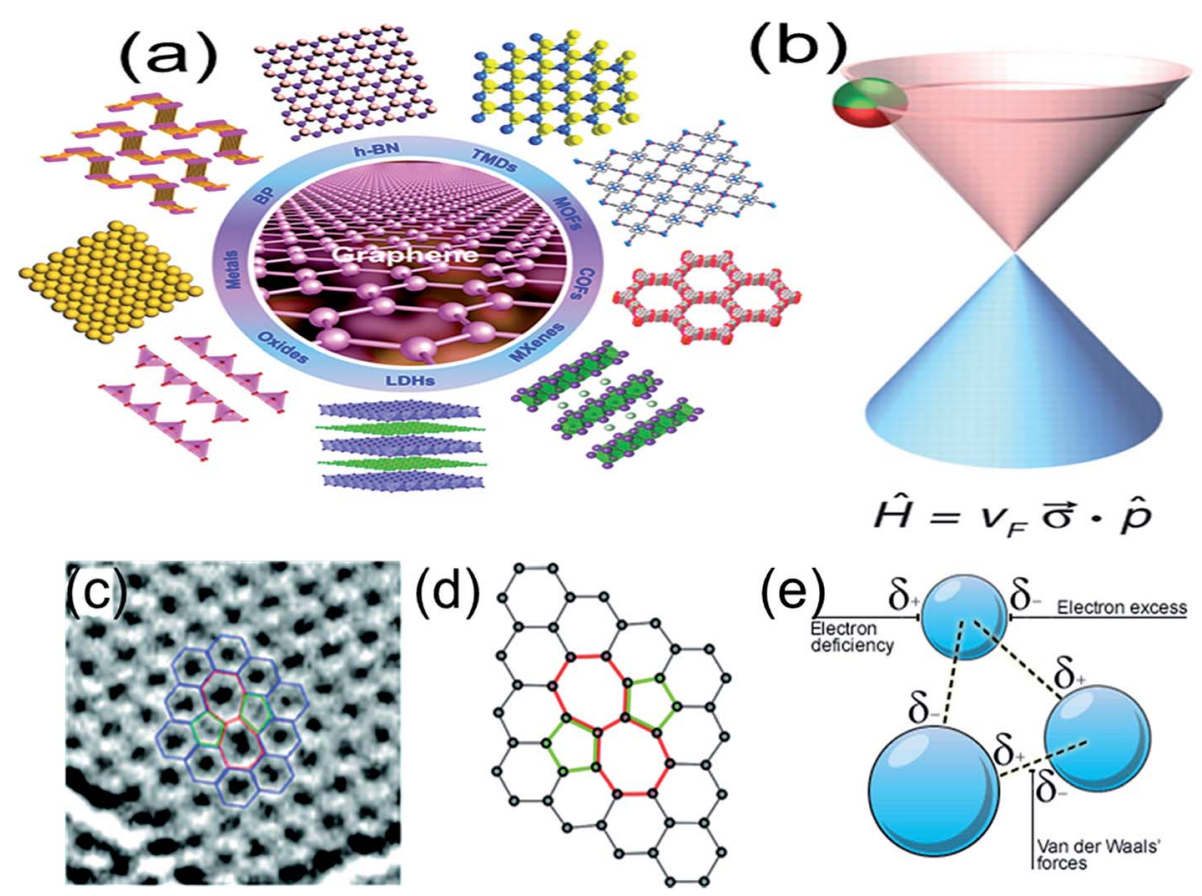

Fig. 1 (a) Schematic illustration of different kinds of typical ultrathin 2D nanomaterials, such as graphene, h-BN, TMDs, MOFs, COFs, MXenes, LDHs, oxides, metals, and BP. Reprinted with permission from ref. 3. Copyright 2015 ACS Publications. (b) Charge carriers in graphene are called massless Dirac fermions and are described by a 2D analog of the Dirac equation, with the Fermi velocity $v_{\mathrm{F}} \approx 1 \times 10^{6} \mathrm{~m} \mathrm{~s} \mathrm{~s}^{-1}$ playing the role of the speed of light and a 2D pseudospin $\vec{\sigma}$ describing two sublattices of the honeycomb lattice. Reprinted with permission from ref. 12 . Copyright 2009 AAAS. (c) TEM image, reprinted with permission from ref. 13. Copyright 2011 ACS Publications. (d) Atomic structure of graphene obtained by DFT calculation. Reprinted with permission from ref. 14. Copyright 2008 ACS Publications. (e) vdW forces in a 2D layered structure. Reprinted from http://bbc.co.uk.

Transistors are the central device building blocks of all modern electronic systems. The first thin film transistors (TFTs) can be dated back to $1962,{ }^{33}$ which used vacuum evaporation technique along with shadow masks to deposit gold electrodes, an n-type polycrystalline cadmium sulfide (CdS) semiconductor channel and a Si monoxide gate insulator on a glass substrate. The integration of TFTs on flexible substrates is of considerable interest for diverse applications including electronic-paper, wearable display and artificial skin, and can enable a new generation of flexible, wearable and disposable electronics. ${ }^{34-37}$ The major challenges for the realization of high performance electronics on flexible substrates are the difficulties in achieving TFTs with a high carrier mobility, high on/off ratio, low operating voltage and using a low temperature-compatible fabrication process.

The highest carrier mobility, excellent optical transparency, high mechanical strength as well as atomic thickness of graphene layers make it extremely useful for flexible FET application. Fabrication of FETs using $2 \mathrm{D}$ graphene crystals as an active component can significantly contribute in the evolution of next generation flexible thin film electronic devices and there have been quite a few reviews summarizing the graphene-based TFTs and their applications. ${ }^{38-45}$ Keeping in view the high demand of graphene based flexible electronics, in this review, we summarize recent development of flexible FETs based on graphene and/or graphene/semiconductor heterojunctions, consistent with the role of graphene as channel layer and/or electrodes in FETs.

\section{Graphene acting as channel material in thin film transistors}

For field-effect transistors (FETs), the level of its carrier mobility often determine the level of some other important performance parameters, such as current density, energy efficiency, switching delay, and so on. High mobility is beneficial to realize the maximum static control, ${ }^{7}$ optical transparency, ${ }^{46}$ sensitivity ${ }^{47}$ and flexibility, ${ }^{48,49}$ and broaden the application fields of TFTs. In this regard, graphene is one of the best choice for transparent conductive thin films in FETs, due to its excellent electronic, mechanical, optical properties and good stability as mentioned above. ${ }^{18,23,29}$

Graphene transparent conductive thin films have been usually used as the conducting channel layer for graphene-based field effect transistors (GFETs). The main opportunities for graphene in both high-performance and low-power applications stem from the atomic thinness achievable in graphene-based devices, which lead to an almost perfect control of the channel potential. Very recently, Johari et al..$^{50}$ have reviewed the electrical performance of GFETs using various architectures and different geometries of graphene conducting channel layer. Graphene has great advantage for its use in flexible electronics but at the same time it needs 
an opening of band gap for TFTs. Researchers had tried to get the good on/off ratio in graphene based FETs by fabricating the graphene as a channel layer. For example, graphene nanoribbon (a kind of graphene with width below $10 \mathrm{~nm}$ (ref. 51)) has been one of the most widely used conducting channel materials for GFETs. Wang et $a .^{52}$ successfully synthesized graphene nanoribbons with smooth edges and size smaller than $10 \mathrm{~nm}$. They demonstrated that the on/off ratio of GFETs increases with the decrease in width of graphene nanoribbon and at room temperature, the on/off ratio and carrier mobility were up to $\sim 1 \times 10^{6}$ and $\sim 200 \mathrm{~cm}^{2}$ $\mathrm{V}^{-1} \mathrm{~s}^{-1}$, respectively. Processing graphene sheets into nanoribbons with widths of less than $10 \mathrm{~nm}$ can open up a bandgap that is large enough for room-temperature transistor operation, but nanoribbon devices often have low driving currents or transconductances. In comparison with graphene nanoribbons, the graphene nanomesh, which can be viewed as many graphene nanoribbons arrange orderly and periodically in another conducting channel material, cannot only introduce the band gap for graphene, but also dramatically increased the driving current of GFET devices. ${ }^{53-55}$ For instance, Bai et al. ${ }^{55}$ successfully obtained the graphene nanomesh with width of $5 \mathrm{~nm}$, on/off ratio of the GFET based graphene nanomesh was about 100, and the current density of GFETs based graphene nanomesh was about 100 times to that of GFETs based on graphene nanoribbons. They prepared the nanomeshes with variable periodicities and neck widths as low as $5 \mathrm{~nm}$ using block copolymer lithography.

\section{Graphene acting as electrode material in thin film transistors}

The conventional electrode of field effect transistors (FETs) is mainly metal or metal oxide with limited applications as a result of their disadvantages such as insensitivity, opacity, non-flexibility as well as large contact resistance between the metal electrode and the active layer of FETs. However, graphene electrode effectively overcomes the above disadvantages of the metal electrode for FETs. Therefore, graphene transparent conductive films are widely used as an electrode material for GFETs, ${ }^{56-62}$ with a heterojunction structure between graphene electrode and the inorganic and/or organic semiconductor active layer.

Heterojunctions refer to an interface between two different semiconductors (electron donor and electron acceptor), where the electronic band structure near the interface is changed according to electrostatics. ${ }^{63}$ The semiconductor heterojunctions have been applied in many solid-state devices, such as solar cells, photo detectors, semiconductor lasers, and lightemitting diodes (LEDs). In 1960, Anderson $^{63}$ successfully prepared high quality heterojunctions for the first time, and provided a detailed energy band diagram and theoretical models. Due to the great influence of the semiconductor junction in our daily life, Nobel Prize in physics was awarded to Zhores I. Alferov, Herbert Kroemer and Jack S. Kilby for their contributions towards the development of semiconductor heterostructures. ${ }^{64-66}$ The weak van der Waals (vdW) interlayer forces in $2 \mathrm{D}$ materials allow us to isolate $2 \mathrm{D}$ monolayer and restack them into arbitrary stacking heterojunctions without

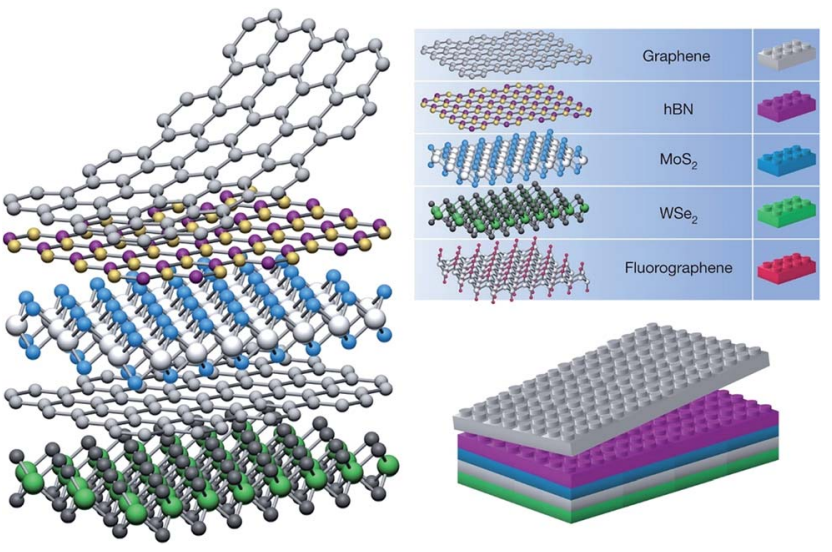

Fig. 2 Schematic 2D heterostructures based on van der Waals interlayer force. Reprinted with permission from ref. 67. Copyright 2013 Nature Publishing Group.

the need to consider the atomic commensurability in contrast to their bulk counterparts. Hence, a new research field of heterojunctions formed by $2 \mathrm{D}$ layered materials has emerged since $2013 .^{67}$ The vertically stacked $2 \mathrm{D}$ layers can be formed by mechanical stacking, serving as a quick and convenient way of forming heterostructures (Fig. 2). In comparison with the horizontal (lateral) heterojunction, the vertical heterojunctions usually have higher electron transition efficiency due to their larger bonding area between two different heterostructured components.

\subsection{Preparation of graphene/semiconductor heterojunctions}

The semiconductor heterojunctions play an important role in modern electronics, being used as an essential building block of solid state devices, such as photo sensors, solar cells, lightemitting diodes and semiconductor lasers. Two-dimensional (2D) materials have the advantages of possessing unique optical bandgap structures and large surface area. Owing to the layered structures of 2D materials, the formation of heterostructures can be in vertical or lateral fashion. Depending on the architecture of the heterostructures, the fabrication methods vary. Here, we sort the fabrication processes into two categories, one is the mechanical stacking method and the other is the direct synthesis.

3.1.1 Mechanical stacking method. Owing to the invention of exfoliation and transfer methods of layered materials, ${ }^{1}$ the mechanically or chemically exfoliated 2D flakes can be manually stacked to form 2D heterostructures, where the interlayer vdW forces hold the heterostructure securely. The 2D flakes can be mechanically/chemically exfoliated from bulk materials or isolated from the synthetic $2 \mathrm{D}$ layers on substrates. The stacking order and the interface of the heterojunctions are critical for their electrical and optical properties. The possibility to form various stacking lattice orientations provides the heterojunction interface with tunable physical properties depending on the interaction strength of the two layered materials. For example, the carrier mobility of graphene on $\mathrm{SiO}_{2}$ is normally limited by the scattering effect of charged impurities, substrate 
Table 1 2D graphene/inorganic semiconductor heterojunctions of thin film transistors ${ }^{a}$

\begin{tabular}{|c|c|c|c|c|}
\hline Year & Layer structure & Mobility $/ \mathrm{cm}^{2} \mathrm{~V}^{-1} \mathrm{~s}^{-1}$ & On/off ratio & Reference \\
\hline 2010 & Graphene/h-BN & $6 \times 10^{4}$ & - & 19 \\
\hline 2012 & h-BN/graphene/h-BN/graphene/h-BN & - & $1 \times 10^{4}$ & 78 \\
\hline 2013 & Graphene/h-BN/MoS 2 & $\sim 45$ & $10^{4}$ to $10^{6}$ & 8 \\
\hline 2013 & Graphene/WS 2 /graphene/h-BN & - & $>1 \times 10^{6}$ & 76 \\
\hline 2013 & Graphene/MoS & - & $>10^{3}$ & 73 \\
\hline 2015 & h-BN/graphene/h-BN & $3 \times 10^{4}$ to $3.6 \times 10^{4}$ & - & 74 \\
\hline 2016 & Graphene/BP & - & $\sim 800$ & 72 \\
\hline
\end{tabular}

surface roughness and $\mathrm{SiO}_{2}$ optical phonons. Dean et al. ${ }^{19}$ have demonstrated a graphene device on h-BN interface layers by using mechanical exfoliated and transfer method. The atomic flat and nearly free charge trapping of h-BN layers serves as excellent substrate for graphene. The carrier mobility of graphene devices fabricated on h-BN substrates is nearly an order higher than the devices on amorphous $\mathrm{SiO}_{2}$ substrates. The graphene layers on h-BN show reduced roughness, less doping and improved chemical stability, demonstrating the critical role of the interfaces. Although the exfoliation technique can produce high quality 2D crystals for fundamental study, it still remains challenging to control the location, layer number and interface of the produced heterojunction, which hinders the practical fabrication.

3.1.2 Direct CVD growth of 2D nanomaterials heterostructures. Although, mechanical stacking serves as a quick and convenient way of forming vertically stacked $2 \mathrm{D}$ heterostructures, the chemical vapor deposition (CVD) method has emerged as a promising method for fabricating $2 \mathrm{D}$ heterojunction in the past few years due to its advantages of being suitable for assembling large area and great variety of 2D stacking structures directly. ${ }^{63}$ The synthesized heterojunction can provide a much cleaner interface for fundamental research and hence better device performance. Furthermore, the development of this methodology has shown the possibility to synthesize lateral 2D heterojunction, which goes beyond the limitations of the mechanical stacking method. It also allows the direct synthesis of various 2D heterojunctions with vertically stacked or laterally stitched interface. For example, Shi et al. ${ }^{68}$ showed that $\mathrm{MoS}_{2}$ can be deposited on a CVDgraphene film via the van der Waals epitaxial growth, through the adsorption and decomposition of $\left(\mathrm{NH}_{4}\right)_{2} \mathrm{MoS}_{4}$ vapor on graphene. The direct formation of the graphene/ $\mathrm{MoS}_{2}$ hybrid film might have some applications based on the highly conductive and transparent graphene electrode coupled with $\mathrm{MoS}_{2}$ as catalyst or active redox centers.

\subsection{Graphene/inorganic semiconductor heterojunction TFT}

Each layer in graphene consists of a covalently bonded, dangling-bond-free lattice and is weakly bound to neighboring layers by van der Waals interactions. This makes it feasible to isolate, mix and match highly disparate atomic layers to create

a wide range of van der Waals heterostructures (vdWHs) without the constraints of lattice matching and processing compatibility. ${ }^{69}$ Since the discovery of graphene, it became clear that when a graphene nanosheet is combined with some inorganic semiconductors, the zero-gap shortcomings, that limit the on/off current ratio of graphene-based transistors, could be considerably reduced or even avoided.

Graphene/inorganic semiconductor heterojunctions could be classified as binary ${ }^{19,70-74}$ and ternary ${ }^{8,75,76}$ heterostructures according to the components in the heterostructures (Table 1). Graphene-based binary heterojunctions usually include graphene/h-BN, ${ }^{19,77}$ graphene $/ \mathrm{MoS}_{2}{ }^{73}$ graphene $/ \mathrm{WS}_{2},{ }^{70}$ graphene/ $\mathrm{BP},{ }^{72}$ and so on. For example, Yu et al. ${ }^{73}$ reported the vertical integration of graphene/ $\mathrm{MoS}_{2}$ heterostructures of layered materials for the fabrication of a new generation of vertical field-effect transistors (VFETs) with a room temperature on/off current ratio $>10^{3}$ and a high current density of up to $5000 \mathrm{~A} \mathrm{~cm}^{-2}$ (Fig. 3a and b). Subsequently, Moriya et al. ${ }^{71}$ further improved the on/off ratio and current density of the VFETs based on graphene/MoS 2 heterostructures to $10^{5}$ and $10^{4} \mathrm{~A} \mathrm{~cm}^{-2}$, respectively (Fig. $3 \mathrm{c}$ and e).

Very recently, Kang et al. ${ }^{72}$ presented a vertical field-effect transistor geometry based on a graphene/black phosphorus

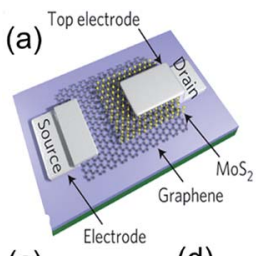

(c)
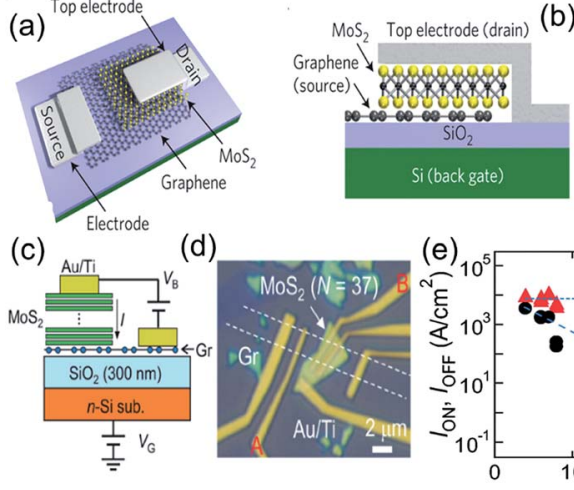

(b)
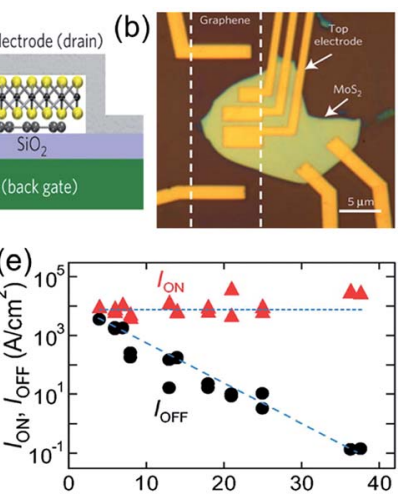

Fig. 3 Schematic illustration ( $a$ and $c$ ) and optical images ( $b$ and $d$ ) of the vertically stacked graphene/MoS 2 heterostructured transistors. (e) The $\mathrm{MoS}_{2}$ layer number dependence of the $I_{\text {on }}$ (current density at $V_{\mathrm{B}}=$ $+0.5 \mathrm{~V}$ and $V_{\mathrm{G}}=+50 \mathrm{~V}$ ) and $I_{\text {off }}$ (current density at $V_{\mathrm{B}}=+0.5 \mathrm{~V}$ and $V_{G}$ $=-50 \mathrm{~V}$ ). Reprinted with permission from ref. 71 and 73. Copyright 2014 AIP Publishing LLC and 2013 Nature Publishing Group. 
(a)

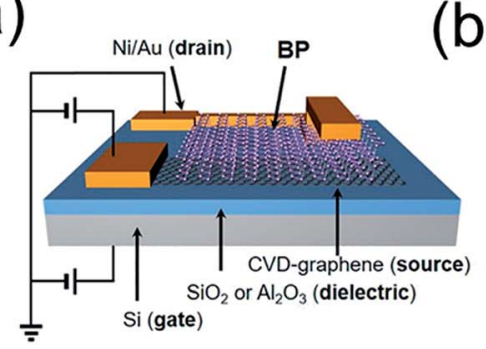

(b)

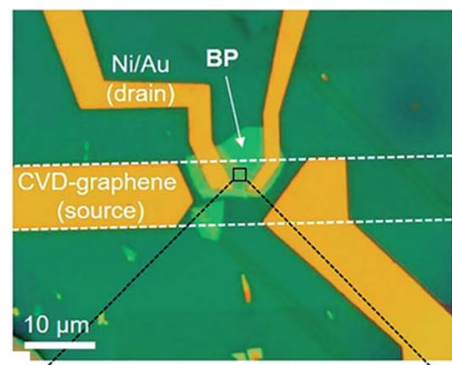

(d)

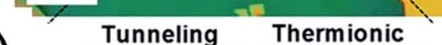

(c)

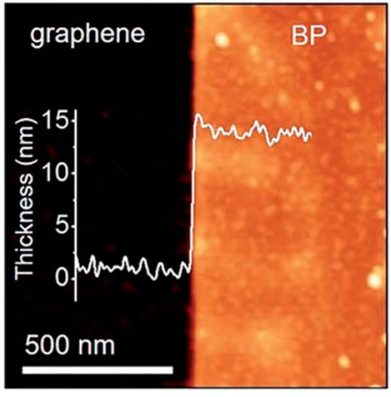
$30 \mathrm{~nm}$

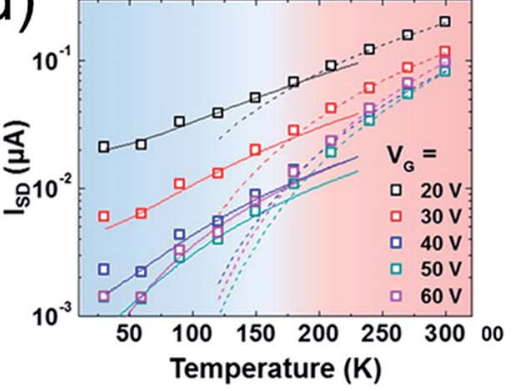

Fig. 4 Schematic illustration (a) and optical microscopy image (b) of the BP-VFET, (c) AFM image at the edge of the BP flake. Inset: crosssectional height profile of the $\mathrm{BP}$ flake on the graphene/SiO$/ 2 / \mathrm{Si}$ substrate, (d) temperature dependence of ISD at different gate voltages from 20 to $60 \mathrm{~V}$ in $10 \mathrm{~V}$ steps. Reprinted with permission from ref. 72. Copyright 2016 ACS.

(graphene/BP) van der Waals heterostructure (Fig. 4). The resulting device characteristics include high on-state current densities $\left(>1600 \mathrm{~A} \mathrm{~cm}^{-2}\right.$ ) and current on/off ratios exceeding 800 at low temperature. Two distinct charge transport mechanisms were identified, which are dominant for different regimes of temperature and gate voltage. In particular, the Schottky barrier between graphene and BP determines charge transport at high temperatures and positive gate voltages, whereas tunneling dominates at low temperatures and negative gate voltages.
Some graphene-based ternary heterojunctions, such as graphene/h-BN/MoS $\left(\right.$ ref. 75) and graphene/h-BN/WS ${ }_{2},{ }^{76}$ have also been reported. For example, Roy et al. ${ }^{75}$ successfully prepared a graphene/h-BN/ $\mathrm{MoS}_{2}$ heterostructured TFT in which graphene was used as the electrode and contacts material and $\mathrm{MoS}_{2} / \mathrm{h}$-BN heterojunction was used as the active layer (Fig. 5). This transistor exhibited n-type behavior with an ON/OFF current ratio of $>10^{6}$, and an electron mobility of $\sim 33 \mathrm{~cm}^{2} \mathrm{~V}^{-1}$ $\mathrm{s}^{-1}$. Uniquely, the mobility does not degrade at high gate

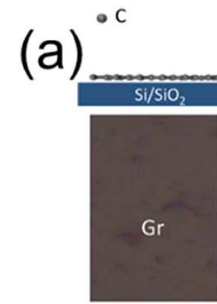

(i)

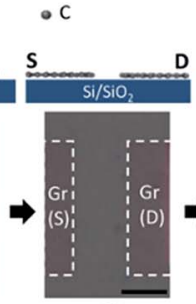

(ii)

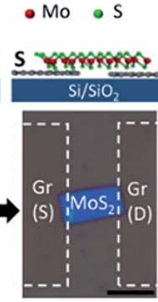

(iii)

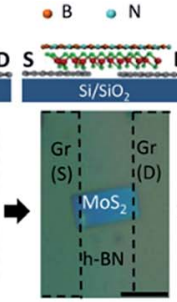

(iv)

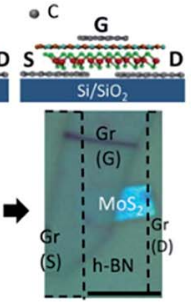

(v)

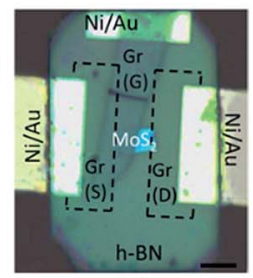

(vi) (b)

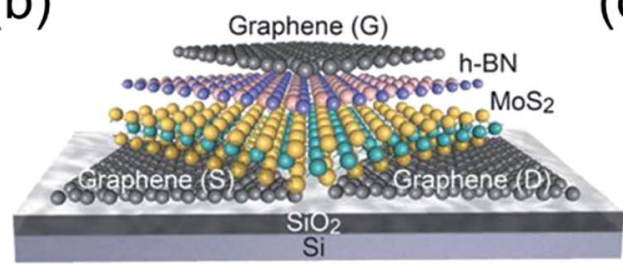

(c)

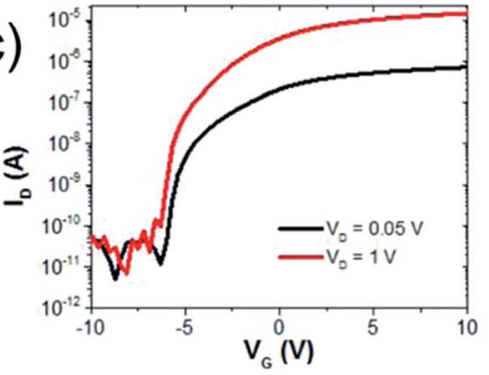

Fig. 5 (a) Schematics (not to scale) of the fabrication process steps (top) and the corresponding optical microscope images (bottom) of a representative graphene/BN/MoS 2 transistor. Scale bar is $5 \mu \mathrm{m}$ for all images. (b and c) Schematic illustration (b) and $I_{D}-I_{G}$ characteristics at different $V_{G}$ (c) of the graphene/BN/MoS 2 transistor. Reprinted with permission from ref. 75. Copyright 2015 ACS. 
(a)
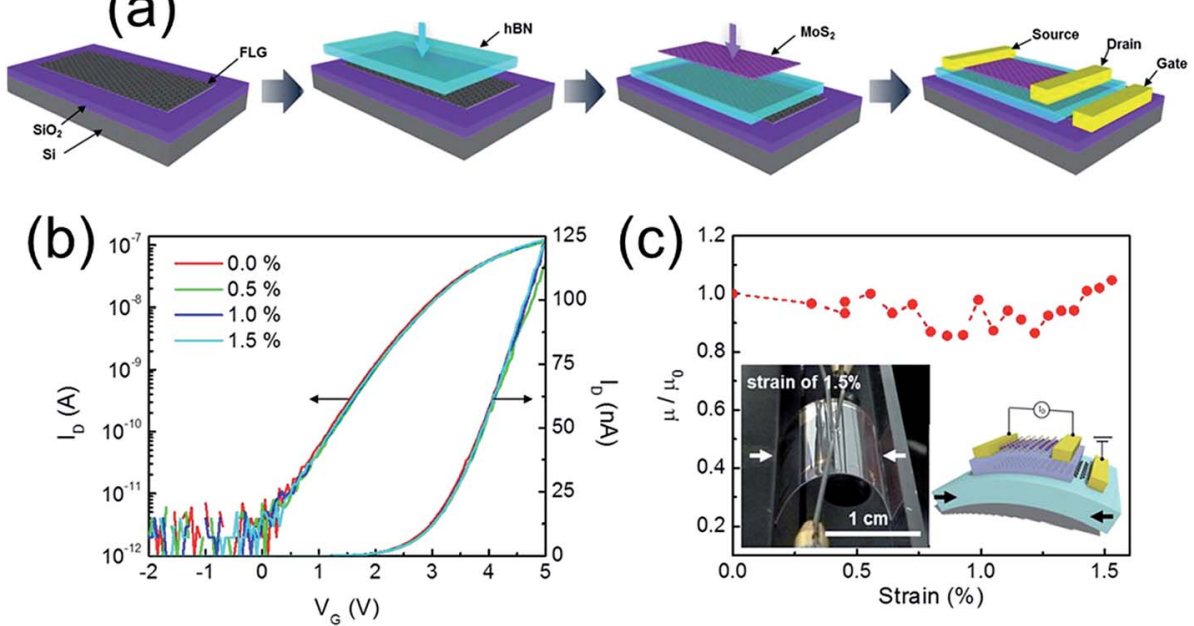

Fig. 6 (a) Schematic of fabrication process for a graphene/BN/MoS 2 transistor (b) transfer curves of the flexible graphene/BN/MoS 2 transistor under different bending conditions up to $1.5 \%$ strain. (c) Relative field-effect mobility of the graphene/BN/MoS 2 transistor as a function of strain. The insets show the photograph of the strained heterostructured transistor by $1.5 \%$ and schematic diagram of the strained device. The arrows in the images indicate the $y$-direction for strain. Reprinted with permission from ref. 8. Copyright 2013 ACS.

Table 2 2D graphene/organic semiconductor heterojunctions of thin film transistors ${ }^{a}$

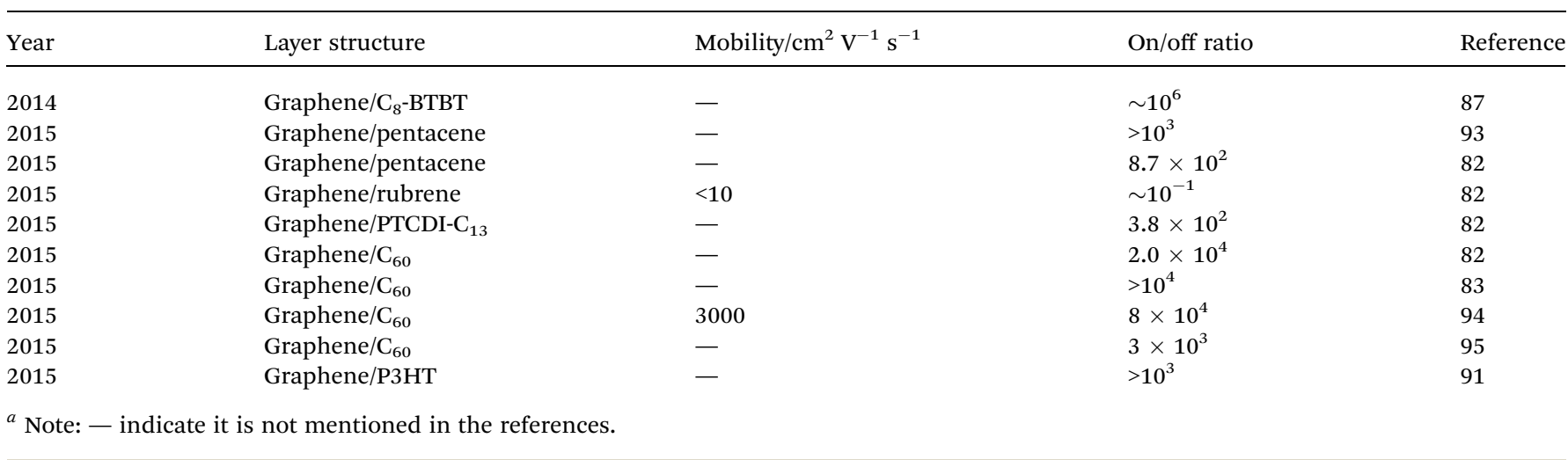

voltages, presenting an important advantage over conventional Si transistors where enhanced surface roughness scattering severely reduces carrier mobilities at high gate-fields.

Lee et $a .^{8}$ also reported a graphene/h-BN/MoS ${ }_{2}$ heterostructured TFT in which graphene was used as the gate electrode whereas $\mathrm{MoS}_{2} / \mathrm{h}-\mathrm{BN}$ heterojunction was used as the active layer (Fig. 6). The high field effect mobility of $45 \mathrm{~cm}^{2} \mathrm{~V}^{-1} \mathrm{~s}^{-1}$ was achieved in trilayer $\mathrm{MoS}_{2}$ at low operating gate voltage $\left(\Delta V_{\mathrm{G}}<10\right.$ $\mathrm{V})$, along with no transport curve hysteresis which is due to the charge-trap-free h-BN dielectric and clean channel/dielectric interface. Moreover, due to the mechanical strength and flexibility of these materials, those FETs show unchanged performance up to $1.5 \%$ applied strain.

\subsection{Graphene/organic semiconductor heterojunction TFT}

Electron injection barrier between the interfaces of graphene and organic materials is low, ${ }^{79}$ which makes graphene/organic hybrids to have good carrier transport behavior and high mobility. Moreover, the work function of graphene is about $4.3 \mathrm{eV}$ and matches the HOMO energy level of many organic semiconductors. ${ }^{\mathbf{8 0 , 8 1}}$ These are the two main reasons why vertical thin film transistors based on graphene/organic semiconductor heterojunction could obtain excellent optoelectronic properties. Graphene is usually used as electron acceptor (ntype), and the electron donor (p-type) in 2D graphene/organic semiconductor heterojunctions mainly consisting of pentacene, ${ }^{82-84}$ rubrene, ${ }^{82,85,86} \mathrm{C}_{8}$-BTBT, ${ }^{87,88}$ PQT-12, ${ }^{89,90}$ P3HT ${ }^{91,92}$ and so on (Table 2).

Shih et $a{ }^{82}$ synthesized a series of graphene/organic semiconductor heterostructured (e.g. graphene/pentacene, graphene $/ \mathrm{C}_{60}$ ) vertical thin film transistors (VFETs) with good optoelectronic properties (Fig. 7 and Table 2). Interestingly, they reported the first experimental evidence that the underlying mechanism of partially screened field effect and selective carrier injection through graphene dominate the electronic transport at the organic semiconductor/graphene heterointerface. They demonstrated flexible organic/graphene VFETs with bending 

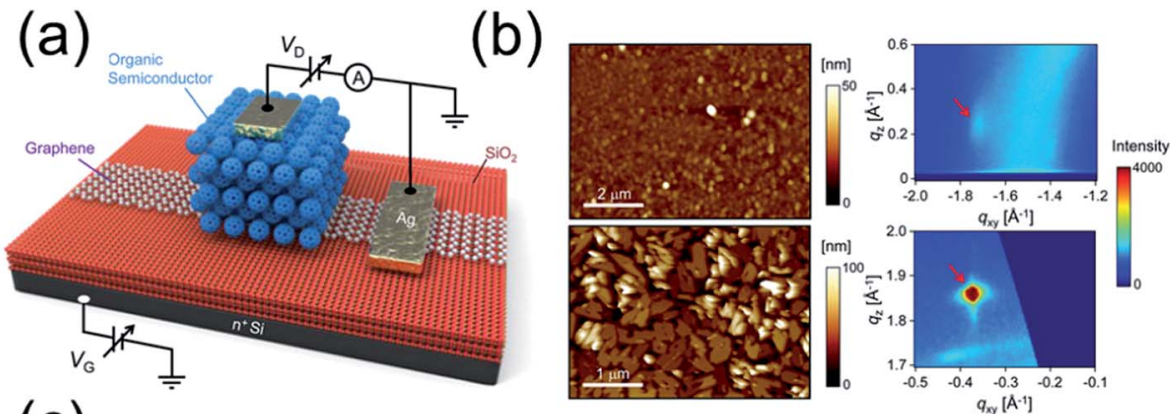

(c)

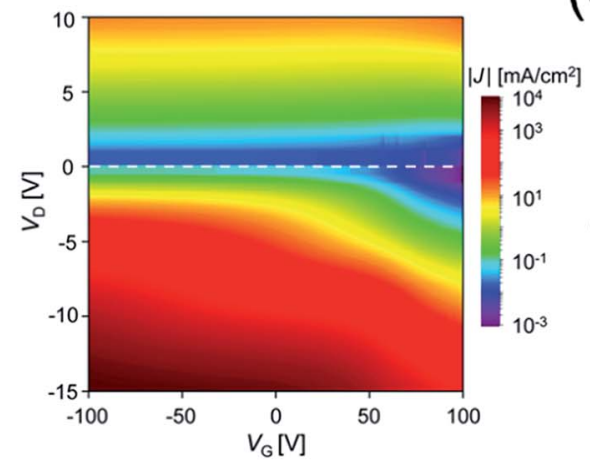

(d)

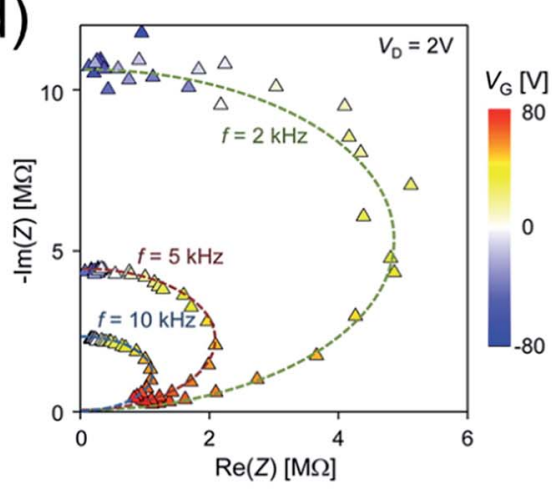

Fig. 7 (a) Schematic of graphene/organic semiconductor heterojunction VFET device. The black connections are associated with the setup for

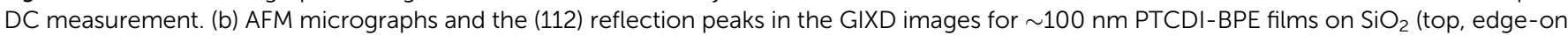
phase) and graphene (bottom, face-on phase). (c) Transport color maps of $\mathrm{J}$ as a function of $V_{\mathrm{D}}$ and $V_{\mathrm{G}}$ for the pentacene $V F E T s$ on as-received $\mathrm{SiO}_{2}$ substrate. (d) Trajectory of $\mathrm{C}_{60}$ impedance at fixed frequencies. By increasing $V_{G}$, the $\mathrm{C}_{60}$ layer is experiencing an insulator-conductor transition, moving from a pure dielectric towards a pure conductor. Reprinted with permission from ref. 82 . Copyright 2015 ACS.

radius $<1 \mathrm{~mm}$ with the output current per unit area equivalent to that of the best oxide planar FETs.

As shown in Fig. 8, Hlaing et al. ${ }^{83}$ reported organic thin film transistors based on vertical heterojunctions of graphene and organic semiconductors (pentacene and $\mathrm{C}_{60}$ ). In these thin heterostructure devices, current modulation is accomplished by tuning the injection barriers at the semiconductor/graphene interface with the application of gate voltage. $\mathrm{N}$-Channel devices fabricated with a thin layer of $\mathrm{C}_{60}$ show a room temperature on/off ratio $>10^{4}$ and current density of up to $44 \mathrm{~mA}$ $\mathrm{cm}^{-2}$. Because of the intrinsic ultra-short channel in the vertical structure, the device is fully operational at a driving voltage of $200 \mathrm{mV}$

Kim et al. ${ }^{93}$ demonstrated a flexible vertical Schottky Barrier (SB) transistor architecture based on a graphene-organic heterojunction and an ion gel gate dielectric. Specifically, an ion gel gate dielectric consisting of an ionic liquid within a network polymer was used to modulate the SB formed at the grapheneorganic (p-type pentacene or n-type $N, N^{\prime}$-dioctyl-3,4,9,10perylenedicarboximide) heterojunction upon application of a bias at the gate located parallel to the source electrode. The resulting $\mathrm{p}$ - and n-type devices could be successfully operated at voltages below $1 \mathrm{~V}$ while yielding a high current density in excess of $100 \mathrm{~mA} \mathrm{~cm} \mathrm{~cm}^{-2}$ and a high on/off current ratio greater than $10^{3}$.

Liu et al. ${ }^{91}$ fabricated high-performance organic vertical thin film transistors (OVTFTs) based on graphene/organic

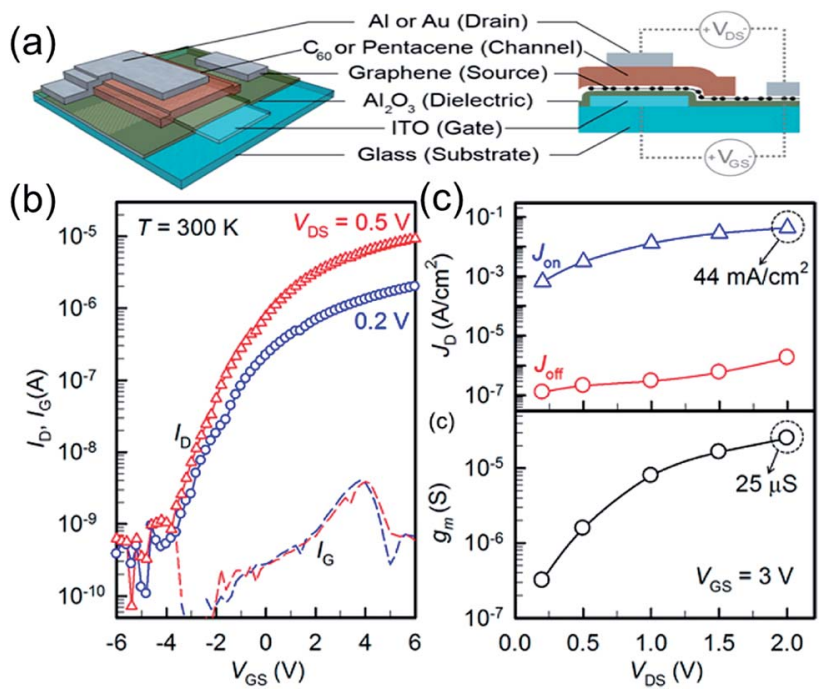

Fig. 8 (a) Schematic illustration of the three-dimensional and crosssectional view of the vertically stacked graphene-organic semiconductor-metal VOTFT. (b) Semi-logarithmic $I_{D}-V_{G S}$ transfer characteristics of an n-type VOTFT measured at $V_{\text {ds }}$ values of 0.2 and $0.5 \mathrm{~V}$. $I_{G}$ is indicated by the dashed line. (c) On- and off-current density (top) and transconductance (bottom) at various drain voltages measured at fixed gate voltage of $3 \mathrm{~V}$. The active area of the transistor measured is 3 $\times 10^{-3} \mathrm{~cm}^{2}$. Reprinted with permission from ref. 83. Copyright 2015 ACS. 

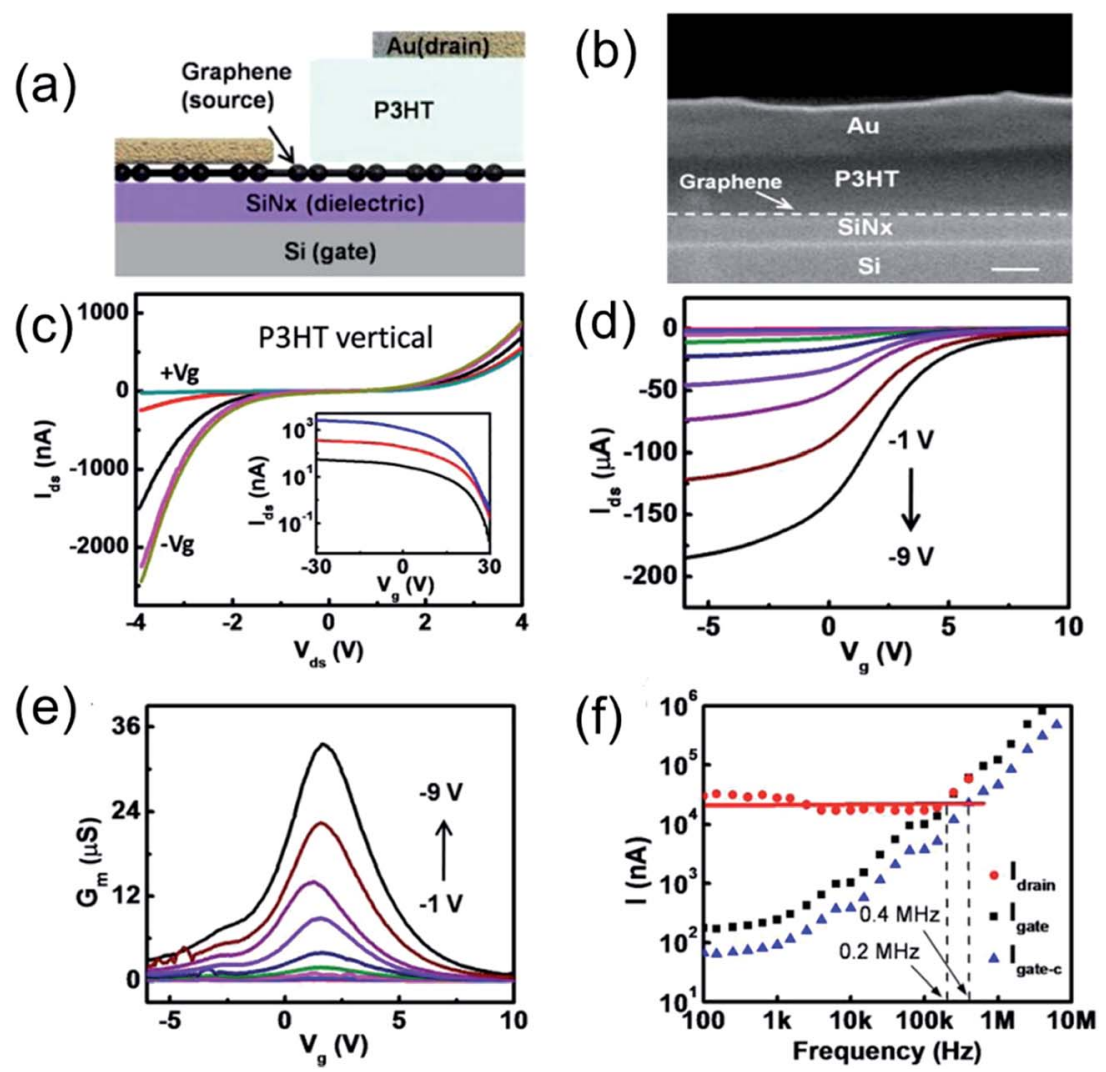

Fig. 9 (a) Schematic illustration of the cross-sectional view of the OVTFT device (b) cross-sectional SEM image of an OVTFT, clearly illustrating layers of the vertical stack. The scale bar is $100 \mathrm{~nm}$. (c) $I_{d s}-V_{d s}$ output characteristics of a graphene-P3HT-Au p-type OVTFTs at various gate voltages from -30 to $+30 \mathrm{~V}$ (15 V steps). The transfer curve is semilog-plotted in the insets with various bias voltages where $V_{\mathrm{ds}}=-1 \mathrm{~V}$ (blue), $-2 \vee($ red), and $-4 \mathrm{~V}$ (black). (d) Transfer characteristics of an OVTFT on glass at different bias voltages from -1 to $-9 \mathrm{~V}(1 \mathrm{~V}$ steps). (e) Transconductance as a function of the gate voltage at different bias voltages. (f) Gate and drain AC current as a function of input frequency signal. The red dot is the measured drain current, and the black square is the measured gate current. The blue triangle is the calibrated gate current by considering the effective gate area and gate capacitance. Reprinted with permission from ref. 91. Copyright 2015 ACS.

semiconducting (P3HT, PBT7 and PCBM) thin film heterostructures through a general strategy of OVTFT fabrication with ultra-short channel length without using conventional highresolution lithography processes (Fig. 9). Electrical studies show that those vertical transistors exhibit excellent switching behavior with an ON/OFF ratio greater than $10^{3}$ and a high current density greater than $3.4 \mathrm{~A} \mathrm{~cm}^{-2}$, and thus enable high cutoff frequency devices comparable to planar ultra-short channel organic transistors.

\section{Conclusions and outlook}

In this review, we have attempted to summarize recent development of flexible thin film transistors (TFT) based on graphene and graphene/semiconductor heterostructures according to the role of graphene which act as electrodes material as well as a channel layer in FET. Especially, we reviewed the progress of TFTs based on graphene/semiconductor (including inorganic and organic semiconductors) heterojunctions in which graphene act as electrodes materials in detail. It must be recognized that there are many caveats and challenges. For example, although the channel material itself may be atomically thin, electric field lines can extend from the drain to the source through the underlying buried oxide, leading to drain-induced barrier lowering, or through the high- $k$ gate dielectric, leading to so-called fringing-field-induced barrier lowering. On the other hand, there are still many challenges ahead in creating large-area and very flat $(<0.2 \mathrm{~nm}$ surface roughness) 2D films, although graphene single crystals up to $5 \mathrm{~cm}$ in diameter have been grown on hydrogen-terminated germanium..${ }^{96}$ Certainly, graphene needs various technological issues to overcome, nevertheless it looks promising for flexible electronic applications, which are difficult to realize with conventional materials. In future, large-area integration of $2 \mathrm{D}$ materials, specifically graphene, with materials of other dimensionality is likely to have a sizable impact on semiconductor technologies. Furthermore, single-crystalline film quality and thickness uniformity with minimal cracks, voids, wrinkles, ripples and other contaminants are critical. Therefore, direct synthesis of highquality 2D materials on insulating substrates should be a high priority. Overall, the vast integration possibilities presented by mixed-dimensional vdW heterostructures suggest considerable future growth potential for this field in both fundamental studies 
and applied technologies. It is anticipated that further progress in 2D material growth, heterostructure formation and device fabrication shall lead to practical applications in future.

\section{Acknowledgements}

This work was supported by Natural National Science Foundation of China and Pakistan Science Foundation Collaborative research project (5161101159), Guangdong Talents Project, NSFC $(51373075,61136003)$ National Basic Research Program of China (973 Program, No. 2015CB856505; 2015CB93220), Natural Science Foundation of Jiangsu Province (BM2012010), the Shenzhen Peacock Program (KQTD2014062714543296) and Shenzhen Key Laboratory of Shenzhen Science and Technology Plan (ZDSYS20140509094114164).

\section{References}

1 K. S. Novoselov, A. K. Geim, S. V. Morozov, D. Jiang, Y. Zhang, S. V. Dubonos, I. V. Grigorieva and A. A. Firsov, Science, 2004, 306(5696), 666-669.

2 G. R. Bhimanapati, Z. Lin, V. Meunier, Y. Jung, J. Cha, S. Das, D. Xiao, Y. Son, M. S. Strano, V. R. Cooper, L. Liang, S. G. Louie, E. Ringe, W. Zhou, S. S. Kim, R. R. Naik, B. G. Sumpter, H. Terrones, F. Xia, Y. Wang, J. Zhu, D. Akinwande, N. Alem, J. A. Schuller, R. E. Schaak, M. Terrones and J. A. Robinson, ACS Nano, 2015, 9(12), 11509-11539.

3 H. Zhang, ACS Nano, 2015, 9(10), 9451-9469.

4 C. N. R. Rao, H. S. S. R. Matte and U. Maitra, Angew. Chem., Int. Ed., 2013, 52(50), 13162-13185.

5 A. Gupta, T. Sakthivel and S. Seal, Prog. Mater. Sci., 2015, 73, 44-126.

6 I. Meric, C. R. Dean, N. Petrone, L. Wang, J. Hone, P. Kim and K. L. Shepard, Proc. IEEE, 2013, 101(7), 1609-1619.

7 H. Fang, S. Chuang, T. C. Chang, K. Takei, T. Takahashi and A. Javey, Nano Lett., 2012, 12(7), 3788-3792.

8 G.-H. Lee, Y.-J. Yu, X. Cui, N. Petrone, C.-H. Lee, M. S. Choi, D.-Y. Lee, C. Lee, W. J. Yoo, K. Watanabe, T. Taniguchi, C. Nuckolls, P. Kim and J. Hone, ACS Nano, 2013, 7(9), 7931-7936.

9 B. Radisavljevic, A. Radenovic, J. Brivio, V. Giacometti and A. Kis, Nat. Nanotechnol., 2011, 6(3), 147-150.

10 Q. Y. He, Z. Y. Zeng, Z. Y. Yin, H. Li, S. X. Wu, X. Huang and H. Zhang, Small, 2012, 8(19), 2994-2999.

$11 \mathrm{~J} . \mathrm{Pu}, \mathrm{Y}$. Yomogida, K.-K. Liu, L.-J. Li, Y. Iwasa and T. Takenobu, Nano Lett., 2012, 12(8), 4013-4017.

12 A. K. Geim, Science, 2009, 324(5934), 1530-1534.

13 D. Jariwala, T. J. Marks and M. C. Hersam, Nat. Mater., 2017, 16(2), 170-181.

14 J. C. Meyer, C. Kisielowski, R. Erni, M. D. Rossell, M. F. Crommie and A. Zettl, Nano Lett., 2008, 8(11), 3582-3586.

15 A. K. Geim and K. S. Novoselov, Nat. Mater., 2007, 6(3), 183191.

16 K. S. Novoselov, V. I. Fal'ko, L. Colombo, P. R. Gellert, M. G. Schwab and K. Kim, Nature, 2012, 490(7419), 192-200.
17 K. S. Novoselov, A. K. Geim, S. V. Morozov, D. Jiang, M. I. Katsnelson, I. V. Grigorieva, S. V. Dubonos and A. A. Firsov, Nature, 2005, 438(7065), 197-200.

18 K. I. Bolotin, K. J. Sikes, Z. Jiang, M. Klima, G. Fudenberg, J. Hone, P. Kim and H. L. Stormer, Solid State Commun., 2008, 146(9-10), 351-355.

19 C. R. Dean, A. F. Young, I. Meric, C. Lee, L. Wang, S. Sorgenfrei, K. Watanabe, T. Taniguchi, P. Kim, K. L. Shepard and J. Hone, Nat. Nanotechnol., 2010, 5(10), 722-726.

20 J.-H. Chen, C. Jang, S. Xiao, M. Ishigami and M. S. Fuhrer, Nat. Nanotechnol., 2008, 3(4), 206-209.

21 S. Hertel, D. Waldmann, J. Jobst, A. Albert, M. Albrecht, S. Reshanov, A. Schoner, M. Krieger and H. B. Weber, Nat. Commun., 2012, 3, 957.

22 M. F. El-Kady, V. Strong, S. Dubin and R. B. Kaner, Science, 2012, 335(6074), 1326-1330.

23 R. R. Nair, P. Blake, A. N. Grigorenko, K. S. Novoselov, T. J. Booth, T. Stauber, N. M. R. Peres and A. K. Geim, Science, 2008, 320(5881), 1308.

24 J. Lu, J.-x. Yang, J. Wang, A. Lim, S. Wang and K. P. Loh, ACS Nano, 2009, 3(8), 2367-2375.

25 G. Eda and M. Chhowalla, Adv. Mater., 2010, 22(22), 23922415.

26 H. Liu, T. Ye and C. Mao, Angew. Chem., Int. Ed., 2007, 46(34), 6473-6475.

27 C.-H. Lu, H.-H. Yang, C.-L. Zhu, X. Chen and G.-N. Chen, Angew. Chem., Int. Ed., 2009, 48(26), 4785-4787.

28 Y.-R. Chang, H.-Y. Lee, K. Chen, C.-C. Chang, D.-S. Tsai, C.-C. Fu, T.-S. Lim, Y.-K. Tzeng, C.-Y. Fang, C.-C. Han, H.-C. Chang and W. Fann, Nat. Nanotechnol., 2008, 3(5), 284-288.

29 C. Lee, X. Wei, J. W. Kysar and J. Hone, Science, 2008, 321(5887), 385-388.

30 A. A. Balandin, S. Ghosh, W. Bao, I. Calizo, D. Teweldebrhan, F. Miao and C. N. Lau, Nano Lett., 2008, 8(3), 902-907.

31 K. S. Novoselov, Z. Jiang, Y. Zhang, S. V. Morozov, H. L. Stormer, U. Zeitler, J. C. Maan, G. S. Boebinger, P. Kim and A. K. Geim, Science, 2007, 315(5817), 1379.

32 M. I. Katsnelson, K. S. Novoselov and A. K. Geim, Nat. Phys., 2006, 2(9), 620-625.

33 P. K. Weimer, Proc. IRE, 1962, 50(6), 1462-1469.

34 A. Dodabalapur, Mater. Today, 2006, 9(4), 24-30.

35 T. Someya, Y. Kato, T. Sekitani, S. Iba, Y. Noguchi, Y. Murase, H. Kawaguchi and T. Sakurai, Proc. Natl. Acad. Sci. U. S. A., 2005, 102(35), 12321-12325.

36 J. A. Rogers, Z. Bao, K. Baldwin, A. Dodabalapur, B. Crone, V. R. Raju, V. Kuck, H. Katz, K. Amundson, J. Ewing and P. Drzaic, Proc. Natl. Acad. Sci. U. S. A., 2001, 98(9), 48354840 .

37 A. Schlierf, P. Samori and V. Palermo, J. Mater. Chem. C, 2014, 2(17), 3129-3143.

38 B. K. Sharma and J. H. Ahn, Solid-State Electron., 2013, 89, 177-188.

39 D. Akinwande, N. Petrone and J. Hone, Nat. Commun., 2014, $5,5678$. 
40 G. Fiori, F. Bonaccorso, G. Iannaccone, T. Palacios, D. Neumaier, A. Seabaugh, S. K. Banerjee and L. Colombo, Nat. Nanotechnol., 2014, 9(10), 768-779.

41 S. J. Park, O. S. Kwon, S. H. Lee, H. S. Song, T. H. Park and J. Jang, Nano Lett., 2012, 12(10), 5082-5090.

42 C. Sire, F. Ardiaca, S. Lepilliet, J. W. T. Seo, M. C. Hersam, G. Darnbrine, H. Happy and V. Derycke, Nano Lett., 2012, 12(3), 1184-1188.

43 C. Biswas and Y. H. Lee, Adv. Funct. Mater., 2011, 21(20), 3806-3826.

44 Q. Y. He, S. X. Wu, Z. Y. Yin and H. Zhang, Chem. Sci., 2012, 3(6), 1764-1772.

45 B. B. Zhan, C. Li, J. Yang, G. Jenkins, W. Huang and X. C. Dong, Small, 2014, 10(20), 4042-4065.

46 S. Lee, K. Lee, C.-H. Liu, G. S. Kulkarni and Z. Zhong, Nat. Commun., 2012, 3, 1018.

47 Q. H. Wang, K. Kalantar-Zadeh, A. Kis, J. N. Coleman and M. S. Strano, Nat. Nanotechnol., 2012, 7(11), 699-712.

48 J. Lee, T.-J. Ha, H. Li, K. N. Parrish, M. Holt, A. Dodabalapur, R. S. Ruoff and D. Akinwande, ACS Nano, 2013, 7(9), 77447750.

49 R. C. Cooper, C. Lee, C. A. Marianetti, X. Wei, J. Hone and J. W. Kysar, Phys. Rev. B: Condens. Matter Mater. Phys., 2013, 87(3), 035423.

50 Z. Johari and R. Ismail, Sci. Adv. Mater., 2015, 7(10), 20112020.

51 X. Li, X. Wang, L. Zhang, S. Lee and H. Dai, Science, 2008, 319(5867), 1229-1232.

52 X. Wang, Y. Ouyang, X. Li, H. Wang, J. Guo and H. Dai, Phys. Rev. Lett., 2008, 100(20), 1586-1594.

53 S. Berrada, N. Viet Hung, D. Querlioz, J. St-Martin, A. Alarcon, C. Chassat, A. Bournel and P. Dollfus, Appl. Phys. Lett., 2013, 103(18), 183509.

54 I. Jung, H. Y. Jang, J. Moon and S. Park, Nanoscale, 2014, 6(12), 6482-6486.

55 J. Bai, X. Zhong, S. Jiang, Y. Huang and X. Duan, Nat. Nanotechnol., 2010, 5(3), 190-194.

56 Y. Song, W. Fang, R. Brenes and J. Kong, Nano Today, 2015, 10(6), 681-700.

57 C.-a. Di, D. Wei, G. Yu, Y. Liu, Y. Guo and D. Zhu, Adv. Mater., 2008, 20(17), 3289-3293.

58 G. Wang, Y. Kim, M. Choe, T.-W. Kim and T. Lee, Adv. Mater., 2011, 23(6), 755-760.

59 H. Chang, G. Wang, A. Yang, X. Tao, X. Liu, Y. Shen and Z. Zheng, Adv. Funct. Mater., 2010, 20(17), 2893-2902.

60 W. Liu, B. L. Jackson, J. Zhu, C.-Q. Miao, C.-H. Chung, Y.-J. Park, K. Sun, J. Woo and Y.-H. Xie, ACS Nano, 2011, 5(3), 2412.

61 J. Y. Choi, W. Kang, B. Kang, W. Cha, S. K. Son, Y. Yoon, H. Kim, Y. Kang, M. J. Ko, H. J. Son, K. Cho, J. H. Cho and B. Kim, ACS Appl. Mater. Interfaces, 2015, 7(10), 6002-6012.

62 W. H. Lee, J. Park, S. H. Sim, S. B. Jo, K. S. Kim, B. H. Hong and K. Cho, Adv. Mater., 2011, 23(15), 1752-1756.

63 R. L. Anderson, Solid-State Electron., 1962, 5(SEP-O), 341344.
64 Z. I. Alferov, V. M. Andreev, D. Z. Garbuzov, Y. V. Zhilyaev, E. P. Morozov, E. L. Portnoi and V. G. Trofim, Fiz. Tekh. Poluprovodn., 1970, 4(9), 1826-1829.

65 I. Hayashi, M. B. Panish and P. W. Foy, IEEE J. Quantum Electron., 1969, 5(4), 211-212.

66 H. Kressel and H. Nelson, RCA Rev., 1969, 30(1), 106-113.

67 A. K. Geim and I. V. Grigorieva, Nature, 2013, 499(7459), 419425.

68 Y. Shi, W. Zhou, A.-Y. Lu, W. Fang, Y.-H. Lee, A. L. Hsu, S. M. Kim, K. K. Kim, H. Y. Yang, L.-J. Li, J.-C. Idrobo and J. Kong, Nano Lett., 2012, 12(6), 2784-2791.

69 Y. Liu, N. O. Weiss, X. Duan, H.-C. Cheng, Y. Huang and X. Duan, Nat. Rev. Mater., 2016, 16042.

70 H. Tan, Y. Fan, Y. Rong, B. Porter, C. S. Lau, Y. Zhou, Z. He, S. Wang, H. Bhaskaran and J. H. Warner, ACS Appl. Mater. Interfaces, 2016, 8(3), 1644-1652.

71 R. Moriya, T. Yamaguchi, Y. Inoue, S. Morikawa, Y. Sata, S. Masubuchi and T. Machida, Appl. Phys. Lett., 2014, 105(8), 083119.

72 J. Kang, D. Jariwala, C. R. Ryder, S. A. Wells, Y. Choi, E. Hwang, J. H. Cho, T. J. Marks and M. C. Hersam, Nano Lett., 2016, 16(4), 2580-2585.

73 W. J. Yu, Z. Li, H. Zhou, Y. Chen, Y. Wang, Y. Huang and X. Duan, Nat. Mater., 2013, 12(3), 246-252.

74 M. A. Stolyarov, G. Liu, S. L. Rumyantsev, M. Shur and A. A. Balandin, Appl. Phys. Lett., 2015, 107(2), 023106.

75 T. Roy, M. Tosun, J. S. Kang, A. B. Sachid, S. B. Desai, M. Hettick, C. C. Hu and A. Javey, ACS Nano, 2014, 8(6), 6259-6264.

76 T. Georgiou, R. Jalil, B. D. Belle, L. Britnell, R. V. Gorbachev, S. V. Morozov, Y.-J. Kim, A. Gholinia, S. J. Haigh, O. Makarovsky, L. Eaves, L. A. Ponomarenko, A. K. Geim, K. S. Novoselov and A. Mishchenko, Nat. Nanotechnol., 2013, 8(2), 100-103.

77 S. J. Kang, G.-H. Lee, Y.-J. Yu, Y. Zhao, B. Kim, K. Watanabe, T. Taniguchi, J. Hone, P. Kim and C. Nuckolls, Adv. Funct. Mater., 2014, 24(32), 5157-5163.

78 L. Britnell, R. V. Gorbachev, R. Jalil, B. D. Belle, F. Schedin, A. Mishchenko, T. Georgiou, M. I. Katsnelson, L. Eaves, S. V. Morozov, N. M. R. Peres, J. Leist, A. K. Geim, K. S. Novoselov and L. A. Ponomarenko, Science, 2012, 335(6071), 947-950.

79 S. Lee, G. Jo, S.-J. Kang, G. Wang, M. Choe, W. Park, D.-Y. Kim, Y. H. Kahng and T. Lee, Adv. Mater., 2011, 23(1), 100-105.

80 Y. Shi, K. K. Kim, A. Reina, M. Hofmann, L.-J. Li and J. Kong, ACS Nano, 2010, 4(5), 2689-2694.

81 Y.-J. Yu, Y. Zhao, S. Ryu, L. E. Brus, K. S. Kim and P. Kim, Nano Lett., 2009, 9(10), 3430-3434.

82 C.-J. Shih, R. Pfattner, Y.-C. Chiu, N. Liu, T. Lei, D. Kong, Y. Kim, H.-H. Chou, W.-G. Bae and Z. Bao, Nano Lett., 2015, 15(11), 7587-7595.

83 H. Hlaing, C.-H. Kim, F. Carta, C.-Y. Nam, R. A. Barton, N. Petrone, J. Hone and I. Kymissis, Nano Lett., 2015, 15(1), 69-74. 
84 D. Jariwala, S. L. Howell, K.-S. Chen, J. Kang, V. K. Sangwan, S. A. Filippone, R. Turrisi, T. J. Marks, L. J. Lauhon and M. C. Hersam, Nano Lett., 2016, 16(1), 497-503.

85 C.-H. Lee, T. Schiros, E. J. G. Santos, B. Kim, K. G. Yager, S. J. Kang, S. Lee, J. Yu, K. Watanabe, T. Taniguchi, J. Hone, E. Kaxiras, C. Nuckolls and P. Kim, Adv. Mater., 2014, 26(18), 2812-2817.

86 F. Liu, W. L. Chow, X. He, P. Hu, S. Zheng, X. Wang, J. Zhou, Q. Fu, W. Fu, P. Yu, Q. Zeng, H. J. Fan, B. K. Tay, C. Kloc and Z. Liu, Adv. Funct. Mater., 2015, 25(36), 5865-5871.

87 D. He, Y. Zhang, Q. Wu, R. Xu, H. Nan, J. Liu, J. Yao, Z. Wang, S. Yuan, Y. Li, Y. Shi, J. Wang, Z. Ni, L. He, F. Miao, F. Song, H. Xu, K. Watanabe, T. Taniguchi, J.-B. Xu and X. Wang, Nat. Commun., 2014, 5, 5162.

88 D. He, Y. Pan, H. Nan, S. Gu, Z. Yang, B. Wu, X. Luo, B. Xu, Y. Zhang, Y. Li, Z. Ni, B. Wang, J. Zhu, Y. Chai, Y. Shi and X. Wang, Appl. Phys. Lett., 2015, $107(18), 183103$.

89 J. Huang, D. R. Hines, B. J. Jung, M. S. Bronsgeest, A. Tunnell, V. Ballarotto, H. E. Katz, M. S. Fuhrer,
E. D. Williams and J. Cumings, Org. Electron., 2011, 12(9), 1471-1476.

90 C. Derry, Y. Wu, S. Gardner and S. Zhu, ACS Appl. Mater. Interfaces, 2014, 6(22), 20269-20275.

91 Y. Liu, H. Zhou, N. O. Weiss, Y. Huang and X. Duan, ACS Nano, 2015, 9(11), 11102-11108.

92 P.-H. Ho, S.-S. Li, Y.-T. Liou, C.-Y. Wen, Y.-H. Chung and C.-W. Chen, Adv. Mater., 2015, 27(2), 282-287.

93 B. J. Kim, E. Hwang, M. S. Kang and J. H. Cho, Adv. Mater., 2015, 27(39), 5875-5881.

94 S. Parui, L. Pietrobon, D. Ciudad, S. Velez, X. Sun, F. Casanova, P. Stoliar and L. E. Hueso, Adv. Funct. Mater., 2015, 25(20), 2972-2979.

95 K. Kim, T. H. Lee, E. J. G. Santos, P. S. Jo, A. Salleo, Y. Nishi and Z. Bao, ACS Nano, 2015, 9(6), 5922-5928.

96 B. Wu, Y. Zhao, H. Nan, Z. Yang, Y. Zhang, H. Zhao, D. He, Z. Jiang, X. Liu, Y. Li, Y. Shi, Z. Ni, J. Wang, J.-B. Xu and X. Wang, Nano Lett., 2016, 16(6), 3754-3759. 